Cochrane Database of Systematic Reviews

\title{
Combination antimicrobial susceptibility testing for acute exacerbations in chronic infection of Pseudomonas aeruginosa in cystic fibrosis (Review)
}

Waters V, Ratjen F

Waters V, Ratjen F.

Combination antimicrobial susceptibility testing for acute exacerbations in chronic infection of Pseudomonas aeruginosa in cystic fibrosis.

Cochrane Database of Systematic Reviews 2017, Issue 6. Art. No.: CD006961.

DOI: 10.1002/14651858.CD006961.pub4. 
HEADER 1

ABSTRACT

PLAIN LANGUAGE SUMMARY

SUMMARY OF FINDINGS

BACKGROUND

OBJECTIVES

METHODS

RESULTS

Figure 1.

DISCUSSION

AUTHORS' CONCLUSIONS

ACKNOWLEDGEMENTS

REFERENCES

CHARACTERISTICS OF STUDIES

APPENDICES

WHAT'S NEW

HISTORY

CONTRIBUTIONS OF AUTHORS

DECLARATIONS OF INTEREST

SOURCES OF SUPPORT

DIFFERENCES BETWEEN PROTOCOL AND REVIEW

INDEX TERMS

\section{TABLE OF CONTENTS}


[Intervention Review]

\section{Combination antimicrobial susceptibility testing for acute exacerbations in chronic infection of Pseudomonas aeruginosa in cystic fibrosis}

Valerie Waters $^{1}$, Felix Ratjen ${ }^{2}$

1Department of Pediatrics, Division of Infectious Diseases, Hospital for Sick Children, Toronto, Canada. 2Department of Pediatrics, The Hospital for Sick Children, Toronto, Canada

Contact address: Valerie Waters, Department of Pediatrics, Division of Infectious Diseases, Hospital for Sick Children, 555 University Avenue, Toronto, ON, M5G 1X8, Canada. valerie.waters@sickkids.ca.

Editorial group: Cochrane Cystic Fibrosis and Genetic Disorders Group.

Publication status and date: Edited (no change to conclusions), published in Issue 3, 2020.

Citation: Waters V, Ratjen F. Combination antimicrobial susceptibility testing for acute exacerbations in chronic infection of Pseudomonas aeruginosa in cystic fibrosis. Cochrane Database of Systematic Reviews 2017, Issue 6. Art. No.: CD006961. DOI: 10.1002/14651858.CD006961.pub4.

Copyright @ 2020 The Cochrane Collaboration. Published by John Wiley \& Sons, Ltd.

\section{A B S T R A C T}

\section{Background}

Antibiotic therapy for acute pulmonary exacerbations in people with cystic fibrosis is usually chosen based on the results of antimicrobial susceptibility testing of individual drugs. Combination antimicrobial susceptibility testing assesses the efficacy of drug combinations including two or three antibiotics in vitro and can often demonstrate antimicrobial efficacy against bacterial isolates even when individual antibiotics have little or no effect. Therefore, choosing antibiotics based on combination antimicrobial susceptibility testing could potentially improve response to treatment in people with cystic fibrosis with acute exacerbations. This is an updated version of a previously published review.

\section{Objectives}

To compare antibiotic therapy based on conventional antimicrobial susceptibility testing to antibiotic therapy based on combination antimicrobial susceptibility testing in the treatment of acute pulmonary exacerbations in people with cystic fibrosis and chronic infection with Pseudomonas aeruginosa.

\section{Search methods}

We searched the Cochrane Cystic Fibrosis and Genetic Disorders Group Cystic Fibrosis Trials Register which comprises of references identified from comprehensive electronic database searches and handsearches of relevant journals and abstract books of conference proceedings. Date of latest search: 19 December 2016.

We also searched ongoing trials registries. Date of latest search: 08 March 2017.

\section{Selection criteria}

Randomised and quasi-randomised controlled studies of antibiotic therapy based on conventional antimicrobial susceptibility testing compared to antibiotic therapy based on combination antimicrobial susceptibility testing in the treatment of acute pulmonary exacerbations in cystic fibrosis due to chronic infection with Pseudomonas aeruginosa.

\section{Data collection and analysis}

Both authors independently selected studies, assessed their quality and extracted data from eligible studies. Additionally, the authors contacted the study investigators to obtain further information. 


\section{Main results}

The search identified one multicentre study eligible for inclusion in the review. This study prospectively assessed whether the use of multiple combination bactericidal antibiotic testing improved clinical outcomes in participants with acute pulmonary exacerbations of cystic fibrosis who were infected with multiresistant bacteria. A total of 132 participants were randomised in the study. The study investigators provided data specific to the 82 participants who were only infected with Pseudomonas aeruginosa for their primary outcome of time until next pulmonary exacerbation. For participants specifically infected with only Pseudomonas aeruginosa, the hazard ratio of a subsequent exacerbation was 0.82 , favouring the control group ( $95 \%$ confidence interval 0.44 to 1.51$)(P=0.52)$. No further data for any of this review's outcomes specific to participants infected with Pseudomonas aeruginosa were available. The risk of bias for the included study was deemed to be low. The quality of the evidence was moderate for the only outcome providing data solely for individuals with infection due to Pseudomonas aeruginosa. For other outcomes, we were unable to judge the quality of the evidence as no data were available for the relevant subset of participants.

\section{Authors' conclusions}

The current evidence, limited to one study, shows that there is insufficient evidence to determine effect of choosing antibiotics based on combination antimicrobial susceptibility testing compared to choosing antibiotics based on conventional antimicrobial susceptibility testing in the treatment of acute pulmonary exacerbations in people with cystic fibrosis with chronic Pseudomonas aeruginosa infection. A large international and multicentre study is needed to further investigate this issue.

The only study included in the review was published in 2005, and we have not identified any further relevant studies up to March 2017 . We therefore do not plan to update this review until new studies are published.

\section{PLAIN LANGUAGE SUMMARY}

\section{Testing antibiotics in combination for acute infections of Pseudomonas aeruginosa in cystic fibrosis}

\section{Review question}

We reviewed the evidence about the effect of testing antibiotics in combination for acute airway infections in people with long-term (chronic) infection with Pseudomonas aeruginosa.

\section{Background}

The main cause of death in people with cystic fibrosis is chronic lung infection. People with cystic fibrosis now live longer due to the aggressive use of antibiotics to treat lung infections. Traditionally, antibiotics are chosen based on the results of laboratory testing of each antibiotic separately against the bacterium (or bug) that is found in the lungs of the person with cystic fibrosis. Antibiotics tested in combination may work effectively against a bacterium even if not effective when tested alone. However, when choosing antibiotics to treat lung infections caused by Pseudomonas aeruginosa in people with cystic fibrosis, it is unclear whether basing the choice of antibiotics on the results of combination testing is better than basing choice on the results of testing antibiotics separately.

This is an updated version of a previously published review.

\section{Search date}

The evidence is current to: 19 December 2016.

\section{Study characteristics}

The search identified one study that tried to answer this question and was eligible for inclusion in the review. The study enrolled 132 people with cystic fibrosis, most of whom ( 82 people) had acute lung infections with Pseudomonas aeruginosa, and randomly put them into two treatment groups. In the first group two antibiotics were selected following the testing of combinations of antibiotics and in the second group the two antibiotics were chosen after testing individual antibiotics to see how effective the drugs were against the bacterium. The study was run across several centres and assessed the clinical outcomes in the participants after a 14-day course of treatment.

\section{Key results}

The study investigators were only able to provide us with data for those who were infected with Pseudomonas aeruginosa for their main outcome (the time until the next acute lung infection). Choosing antibiotics based on the results of combination antibiotic testing did not lead to a longer time until the next lung infection compared to choosing antibiotics based on results of separate testing. They could not provide us with any results people infected with Pseudomonas aeruginosa for other outcomes in our review.

\section{Quality of the evidence}

We are satisfied that the people taking part were divided into the different treatment groups completely at random and no one could have foreseen which group any individual would be in. We are also satisfied that during the study, neither the individuals or clinic personnel

Combination antimicrobial susceptibility testing for acute exacerbations in chronic infection of Pseudomonas aeruginosa in cystic 
knew which treatment group each individual was in. There were no missing data from the study. The quality of the evidence for the only outcome for which we have data (time to the next lung infection) is moderate, but we could not judge the quality of the evidence for other outcomes as there were no separate results available for people infected with Pseudomonas aeruginosa. 


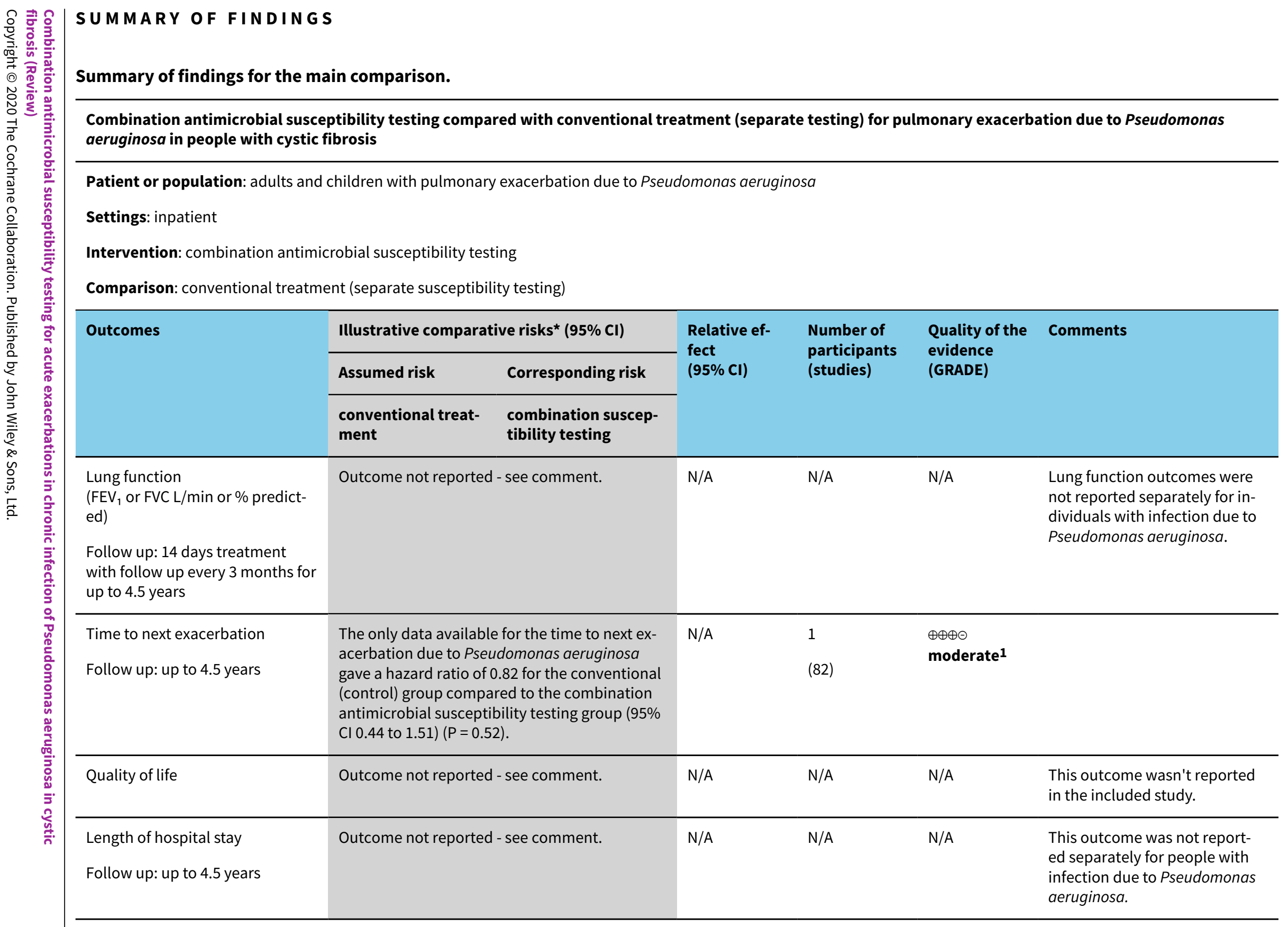




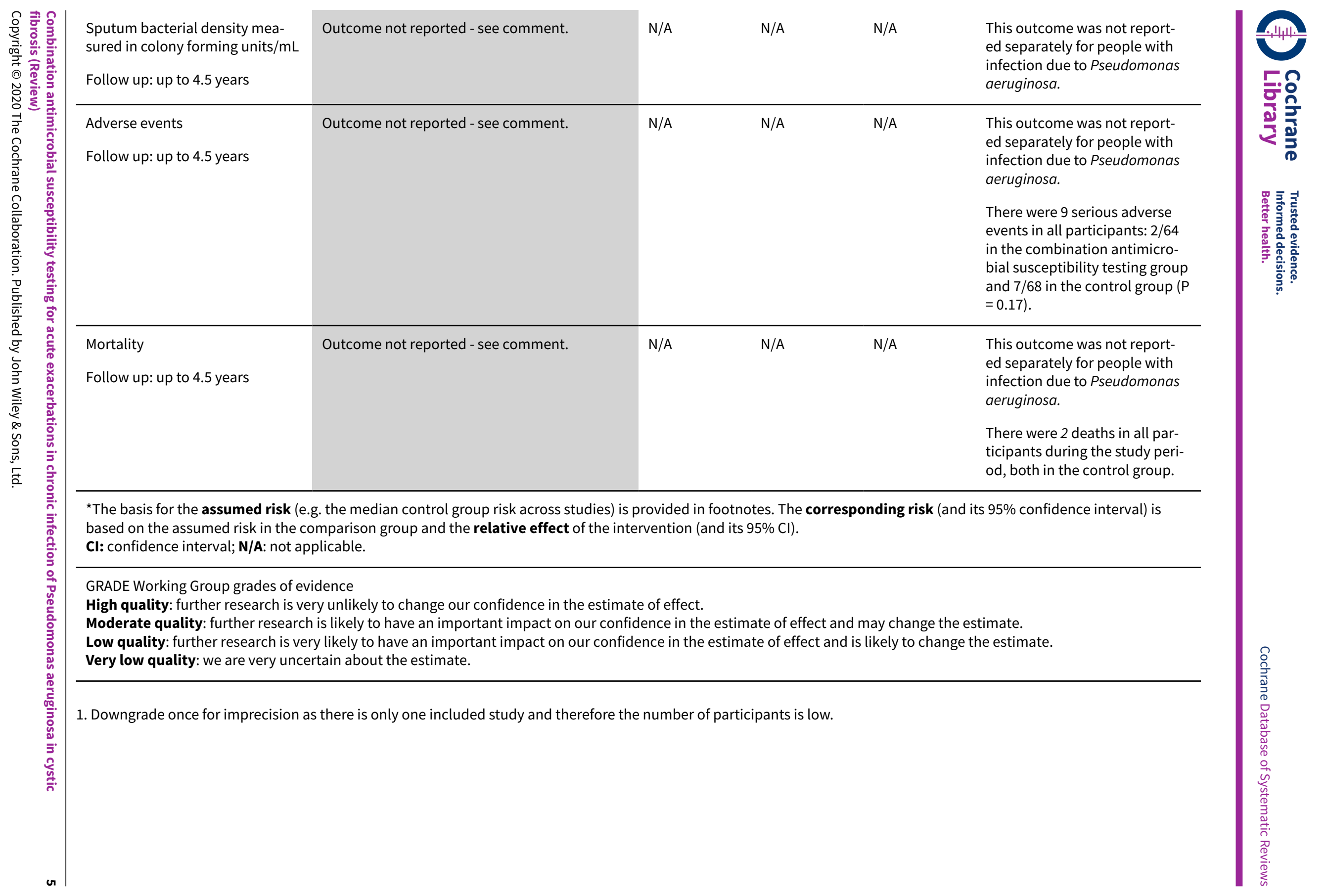




\section{B A C K G R O U N D}

\section{Description of the condition}

Cystic fibrosis (CF) is the most common life-limiting genetic condition in white populations and respiratory failure caused by chronic pulmonary infection is the primary cause of death in people with CF (Gibson 2003). Staphylococcus aureus and Haemophilus influenzae are typically initially detected in respiratory cultures. Over time, people with CF become chronically infected with mucoid Pseudomonas aeruginosa ( $P$ aeruginosa) with associated declining pulmonary function and increasing symptoms (Burns 2001; Henry 1992; Kosorok 2001; Pamukcu 1995).

\section{Description of the intervention}

Over the past several decades, the life expectancy of people with CF has increased significantly, due partly to the aggressive use of antibiotics in the treatment of respiratory infections (Gibson 2003). The standard of care for the treatment of pulmonary exacerbations includes intravenous (IV) antibiotic use. Bacterial strains isolated from the respiratory tract of people with CF are commonly tested for in vitro antimicrobial susceptibility. Conventional in vitro antimicrobial susceptibility testing is done on selected morphotypes, or colony types, of bacteria such as $P$ aeruginosa. However, there may be a significant amount of variability in the antimicrobial susceptibility patterns of different morphotypes and even of individual colonies of the same morphotype of $P$ aeruginosa (Foweraker 2005). Conventional antimicrobial susceptibility testing may thus underestimate resistance and results are often not reproducible. Although in vitro antimicrobial susceptibility testing has been shown to be of benefit in guiding antibiotic choices for pulmonary infections in people who don't have CF, the role of conventional susceptibility testing in guiding the treatment of pulmonary exacerbations in people with CF is less clear (Gaillard 1995; Smith 2003). In fact, clinicians will often ignore the results of susceptibility testing of bacterial strains obtained during a pulmonary exacerbation if the individual is already improving on IV antibiotics.

\section{How the intervention might work}

If conventional antimicrobial susceptibility testing identifies a multi-drug resistant bacterial strain from the respiratory tract of an individual with CF, combination antimicrobial susceptibility testing may be undertaken on the strain. This type of testing often shows that combinations of two or three antibiotics have in vitro activity against a bacterial isolate when individual antibiotics have little or none (Aaron 2000; Saiman 1996). Although combination antibiotic therapy may be better than antibiotic monotherapy to treat CF exacerbations caused by $P$ aeruginosa (Smith 1999), choosing antibiotics based on multiple combination bactericidal antibiotic testing (MCBT), a type of combination antimicrobial susceptibility testing, may not affect clinical outcomes (Aaron 2005).

Both conventional and combination antimicrobial testing are usually done on bacteria growing planktonically or "free-floating". There is growing evidence that bacterium such as $P$ aeruginosa actually grows as a biofilm or a "slime layer" in the airways of people with CF with chronic pulmonary infections (Drenkard 2002; Singh 2000). Biofilms are communities of bacteria embedded in an exopolysaccharide matrix and are highly resistant to killing by antibiotics (Prince 2002). Work has been done to develop antimicrobial susceptibility testing based on biofilm growth of bacteria rather than planktonic growth in an effort to mimic the pathophysiology of the CF lung (Ceri 1999; Moskowitz 2004; Moskowitz 2005). Although biofilm antimicrobial susceptibility testing of $P$ aeruginosa strains has demonstrated different, more resistant susceptibility profiles, the effect of these results on treatment outcomes has yet to be determined.

\section{Why it is important to do this review}

In light of the uncertainties we have described, we aimed to compare antibiotic therapy based on conventional antimicrobial susceptibility testing to antibiotic therapy based on combination antimicrobial susceptibility testing in the treatment of acute pulmonary exacerbations in CF.

This is an updated version of a previously published review (Waters 2008; Waters 2015).

\section{O B J E C T IVES}

The objective was to compare antibiotic therapy based on conventional antimicrobial susceptibility testing to antibiotic therapy based on combination antimicrobial susceptibility testing in the treatment of acute pulmonary exacerbations in people with $C F$ and chronic infection with $P$ aeruginosa.

\section{METHODS}

\section{Criteria for considering studies for this review}

\section{Types of studies}

Randomised (RCTs) and quasi-randomised controlled trials.

\section{Types of participants}

Adults and children (with all levels of disease severity) diagnosed with CF, confirmed with sweat test or genetic testing or both with an acute pulmonary exacerbation due to $P$ aeruginosa.

\section{Types of interventions}

The intervention was antibiotic therapy based on conventional antimicrobial susceptibility testing to antibiotic therapy based on combination antimicrobial susceptibility testing in the treatment of acute pulmonary exacerbations in CF due to chronic infection with Paeruginosa.

An acute pulmonary exacerbation was defined according to symptoms, chest examination findings and change in forced expiratory volume in one second (Rosenfeld 2001). We defined chronic infection as follows: when measured monthly over a 12month period, more than $50 \%$ of months when samples had been taken, were $P$ aeruginosa culture positive (Lee 2003).

\section{Types of outcome measures}

We planned to measure outcomes at less than a week, one to two weeks, more than two weeks to three weeks, more than three weeks to four weeks; the outcome 'Time to next pulmonary exacerbation' would be measured in monthly intervals after these time points. We would have also considered outcomes measured at other time points.

Combination antimicrobial susceptibility testing for acute exacerbations in chronic infection of Pseudomonas aeruginosa in cystic 


\section{Primary outcomes}

1. Lung function

a. forced expiratory volume in one second $\left(\mathrm{FEV}_{1}\right)(\mathrm{L} / \mathrm{min}$ or $\%$ predicted)

b. forced vital capacity (FVC) (L/min or \% predicted)

2. Time to next pulmonary exacerbation

\section{Secondary outcomes}

1. Quality of life

2. Length of hospital stay

3. Sputum bacterial density measured in colony forming units $/ \mathrm{mL}$ (CFU/mL)

4. Adverse events

5. Mortality

\section{Search methods for identification of studies}

There are no restrictions regarding language or publication status.

\section{Electronic searches}

We identified relevant trials from the Group's Cystic Fibrosis Trials Register using the term 'susceptibility testing' OR 'sensitivity testing'.

The Cystic Fibrosis Trials Register is compiled from electronic searches of the Cochrane Central Register of Controlled Trials (CENTRAL) (updated each new issue of the Cochrane Library), weekly searches of MEDLINE, a search of Embase to 1995 and the prospective handsearching of two journals - Pediatric Pulmonology and the Journal of Cystic Fibrosis. Unpublished work is identified by searching the abstract books of three major cystic fibrosis conferences: the International Cystic Fibrosis Conference; the European Cystic Fibrosis Conference and the North American Cystic Fibrosis Conference. For full details of all searching activities for the register, please see the relevant sections of the Cystic Fibrosis and Genetic Disorders Group website.

Date of the most recent search of the Group's Cystic Fibrosis Trials Register: 19 December 2016.

We also checked the National Institutes of Health (NIH) sponsored website www.clinicaltrials.gov and the WHO ICTRP website http:// apps.who.int/trialsearch/ for any ongoing studies with potential interim results. For search terms please see the appendices (Appendix 1; Appendix 2). Date of last search: 08 March 2017.

\section{Searching other resources}

We checked the reference lists of all studies identified for any further relevant studies.

\section{Data collection and analysis}

\section{Selection of studies}

The two authors (VW, FR) independently applied the inclusion criteria to all potential studies. The authors were not blinded to the studies. The authors planned to resolve any disagreements by discussion with a third person (Nikki Jahnke (NJ)).

\section{Data extraction and management}

Using a data collection form, authors VW and FR independently obtained data from published reports or from study investigators. They would have resolved any disagreement by discussion with a third person (Nikki Jahnke (NJ)). In addition to information about study references and authors and verification of study eligibility, the data collection form included information about the methods of the study (e.g. study duration, type of trial, blinding, number of dropouts and potential confounders). The authors also reported characteristics of the study participants including age, sex and setting of the study on the form. The authors described the intervention, specifically antibiotic therapy, with regards to type of antibiotic, route of delivery, doses and length of treatment. The authors collected data for all randomised participants. When possible, the authors planned to record the mean change (before and after antibiotic therapy) in FEV ${ }_{1}$ and FVC, the mean quality of life score after antibiotic therapy, the mean hospital length of stay, the mean change in sputum bacterial density (before and after antibiotic therapy) and the number of adverse events and mortalities. For each mean value, they also planned to obtain the standard deviation. For time to next exacerbation, they planned to collect log-rank estimates and Cox model estimates.

\section{Assessment of risk of bias in included studies}

Authors VW and FR independently assessed the risk of bias of the included study, originally using the following criteria (Jüni 2001), which they later adapted and expanded using the criteria in the Cochrane risk of bias tool

\section{Assessment of generation of allocation sequences}

The authors assessed each study as to the generation of allocation sequences:

1. adequate: if allocation sequence is suitable to prevent selection bias (i.e. random numbers table, drawing envelopes, tossing a coin, throwing dice etc);

2. inadequate: if allocation sequence could be related to prognosis and thus introduce selection bias (i.e. assigning participants based on case record number, date of birth, date of admission etc);

3. unclear: if the study is described as randomised but the method used to generate the allocation sequence is not stated.

\section{Assessment of concealment of allocation sequences}

The authors also assessed the method used to conceal the allocation sequences in each study:

1. adequate: if participants and investigators cannot predict which group the participant will be assigned to (i.e. coded drug containers, central randomisation, numbered, sealed, opaque envelopes etc);

2. inadequate: if participants and investigators can predict which group the participant will be assigned to and thus introduce selection bias (i.e. open allocation schedule, non-opaque envelopes etc);

3. unclear: if the method of concealing the allocation sequence is not described. 


\section{Assessment of blinding}

In order to determine the potential for performance and detection bias, the authors assessed each study with respect to the degree of blinding:

1. the participant is blinded to participant assignment;

2. the care provider is blinded to participant assignment;

3. the investigator measuring study outcomes is blinded to participant assignment.

\section{Assessment of follow up}

To assess for the possibility of attrition bias, the authors examined each study with respect to:

1. whether or not it was stated how many participants were lost to follow up and why they were lost to follow up;

2. whether or not an intention-to-treat analysis was used (i.e. inclusion in the final analysis of all randomised participants into a trial in the groups to which they were randomised irrespective of what happened subsequently).

\section{Incorporating assessments of study validity in reviews}

The authors planned to weigh studies according to the inverse of the variance of the estimated measure of effect. If we considered there was a high risk of bias, we would have investigated the effects of this with a sensitivity analysis (see below).

\section{Measures of treatment effect}

For dichotomous data (adverse events, mortality), the authors planned to gather information on participants randomised to each treatment group, based on an intention-to-treat analysis. They planned to include interim results from individual randomised participants from ongoing studies in the analysis. They defined time points for each study outcome according to when it was measured (less than a week, one to two weeks, more than two weeks to three weeks, more than three weeks to four weeks); they planned to analyse study outcomes separately according to these time points. As the authors identified only one study for inclusion in the review, they did not combine results from different studies. However, for future updates, when they are able to include more studies, they plan to pool the treatment effect across studies to determine an odds ratio with $95 \%$ confidence intervals for each study outcome.

For continuous data ( $\mathrm{FEV}_{1}, \mathrm{FVC}$, quality of life, length of hospital stay, sputum bacterial density), the authors planned to calculate the difference between the mean values (mean difference (MD)) of treatment effect for each group. As a summary statistic across studies, they will use the MD if the same scale is used or the standardised mean difference (SMD) if different scales are used (e.g. quality of life measurements). For time-to-event data (time to next exacerbation), most studies use Kaplan-Meier survival analysis. The authors thus planned to collect log-rank estimates and Cox model estimates to subsequently summarise the time-to-event data as a hazard ratio with 95\% Cls (Higgins 2011; Parmar 1998).

\section{Unit of analysis issues}

If the authors identify cluster-randomised studies in the future, they will include the data if the relevant information is available. The authors will calculate the intracluster correlation coefficient (ICC) according to Donner (Donner 2001).

\section{Dealing with missing data}

Although a true intention-to-treat analysis must include all participants who were randomised, regardless of whether their outcomes were actually collected, in reality, data are often missing for participants who are lost to follow up. This was not the case in the only study included in the review. However, for future updates, the authors will perform an available-case analysis (analysing data for every participant for whom the outcome is obtained) in these situations. The authors will collect the percentages of participants from whom no outcome data were available and will report these on the data collection form. The authors will include data on only those whose results are known, using as a denominator the total number of people who completed the study for the particular outcome in question. The authors will consider the variation in the degree of missing data across studies as a potential source of heterogeneity.

\section{Assessment of heterogeneity}

In performing a meta-analysis, the authors planned to measure the variability of results between trials (heterogeneity) using the $\mathrm{I}^{2}$ statistic described by Higgins (Higgins 2003). The $\mathrm{I}^{2}$ statistic describes the percentage of total variation across studies that is due to heterogeneity rather than by chance. It is calculated using Cochran's heterogeneity statistic and the degrees of freedom. The $I^{2}$ statistic can range from $0 \%$ to $100 \%$, where a value of $0 \%$ indicates no observed heterogeneity and larger values show increasing heterogeneity. A value greater than $50 \%$ may be considered substantial heterogeneity.

\section{Assessment of reporting biases}

As the searches identified only one study which was eligible for inclusion, the authors were not able to assess publication bias. If sufficient studies are included in the future, they will assess publication bias in a future update by constructing a funnel plot. In the absence of bias, the plot should resemble a symmetrical inverted funnel (Higgins 2011). If there is asymmetry, the authors will consider publication bias and other reasons (such as location biases, true heterogeneity, poor methodological quality of smaller studies etc.) as a potential cause.

\section{Data synthesis}

The authors identified only one study for inclusion the review. For future updates, if the authors include more studies, they plan to combine multiple studies as follows. If the studies are too clinically diverse (e.g. different lengths of antibiotic treatment), the authors will not perform a meta-analysis. If the studies are considered clinically similar enough to combine and where there is no significant heterogeneity, they will calculate the pooled effect estimates using a fixed-effect model. If there is statistical heterogeneity, the authors will investigate as outlined below and will perform a random-effects meta-analysis to incorporate heterogeneity among studies.

\section{Subgroup analysis and investigation of heterogeneity}

If the authors find significant heterogeneity (assessment as detailed above and $\mathrm{P}<0.10$ by the $\mathrm{Q}$ test) (Higgins 2011), they will explore the potential causes of this (i.e. different types of antimicrobial susceptibility testing, different participant populations etc.) and if possible, conduct subgroup analyses of the studies. For example, study results may vary if different types of combination

Combination antimicrobial susceptibility testing for acute exacerbations in chronic infection of Pseudomonas aeruginosa in cystic 
antimicrobial susceptibility testing are used (e.g. MCBT compared to checkerboard dilution assays). In addition, results may vary if one study has more adult participants who can produce sputum (a more accurate sample with potentially more reliable susceptibility results) and another study has more pediatric participants who can only do throat swabs (a less reliable respiratory tract sample).

\section{Sensitivity analysis}

When the authors are able to include more studies in the review, they will perform a sensitivity analysis to determine the robustness of the results. They will investigate whether changing which studies are included, based on our assessment of the methodological quality (including or excluding CCTs, including or excluding trials reporting degrees of blinding etc.) or changing our chosen statistical model (i.e. random effects model compared to a fixed-effect model) changes the results of our review. If the sensitivity analysis does not significantly change the results, it strengthens the confidence that can be placed in these results.

\section{Summary of findings table}

In a post hoc change to the protocol we have included a summary of findings table for the comparison of combination antibiotic susceptibility testing with conventional single antibiotic susceptibility testing (Summary of findings for the main comparison).

We included the following outcomes: change in lung function (FEV and $\mathrm{FVC} \%$ predicted and $\mathrm{L} / \mathrm{min}$ ), time to next exacerbation, quality of life, length of hospital stay, sputum bacterial density (measured in $\mathrm{CFU} / \mathrm{mL}$ ), adverse events and mortality.

To determine the quality of the evidence we used the GRADE approach in which the quality of the evidence is downgraded where there was high risk of bias in included trials, indirectness of the evidence to our population of interest, imprecision of the results or a high risk of publication bias. The quality of evidence may be downgraded once if the reason is serious and twice if the reason is deemed to be very serious.

\section{RES U LTS}

\section{Description of studies}

\section{Results of the search}

The searches identified four studies and only one study was eligible for inclusion in the review (Aaron 2005).

\section{Included studies}

The only included study was a multicentre, randomised, doubleblind controlled clinical study that prospectively assessed whether the use of combination antibiotic susceptibility testing improved clinical outcomes in participants with acute pulmonary exacerbations of CF who were infected with multiresistant bacteria. Participants who developed an exacerbation of pulmonary disease were randomised to receive a 14-day course of any two IV antibiotics (labelled "antibiotic \#1" and "antibiotic \#2") chosen on the basis of either results from conventional sputum culture and sensitivity testing or the result of MCBT. Individuals were eligible for enrolment into the study if they had a confirmed diagnosis of CF, were at least 12 years old, could spontaneously produce sputum for culturing and were chronically infected with multiresistant $P$ aeruginosa, Burkholderia cepacia complex, Stenotrophomonas maltophilia or Achromobacter xylosoxidans. Although the study included participants who were infected with different types of multiresistant organisms, the majority were infected with $P$ aeruginosa $(67.2 \%$ in the MCBT group and $57.4 \%$ in the control group). The authors have provided us with the data specific to the participants who were only infected with $P$ aeruginosa for the study's primary outcome; however the information about the study participants given below pertains to the whole study cohort. The primary outcome of the study was time from randomisation until the participant's next pulmonary exacerbation and corresponded to the length of participant follow up. Outcome measurements were taken and reported for day 0 and day 14 of antibiotic treatment.

A total of 132 participants were randomised in the study; 64 to the MCBT-treated group and 68 to the conventionally-treated (control) group. All 132 participants received the intended treatment and all 132 participants were included in the final analysis. The mean age (SD) in the MCBT group was 29.5 years (8.2) and in the control group was 25.8 years (6.5). In the MCBT group, 29 participants were male and 35 were female and in the control group, 31 were male and 37 were female. The baseline FEV ${ }_{1}$ (SD) in the MCBT group was 1.67 $L(0.66)$ and was $1.63 L(0.67)$ in the control group. The number of participants with diabetes was $15(23.4 \%)$ in the MCBT group and $13(19.1 \%)$ in the control group. The number of participants with pancreatic insufficiency was $63(98.4 \%)$ in the MCBT group and 65 $(95.6 \%)$ in the control group. The number of participants with liver disease was $6(9.4 \%)$ in the MCBT group and $8(11.8 \%)$ in the control group.

\section{Excluded studies}

Two studies were excluded as they did not examine combination antimicrobial testing (Oermann 2013; Wainwright 2011). The remaining two studies were excluded as they examined biofilm antimicrobial susceptibility testing (Moskowitz 2011; Waters 2010).

\section{Risk of bias in included studies}

The overall risk of bias was minimal in the included study (Figure 1). 
Figure 1. Risk of bias summary: review authors' judgements about each risk of bias item for each included study.

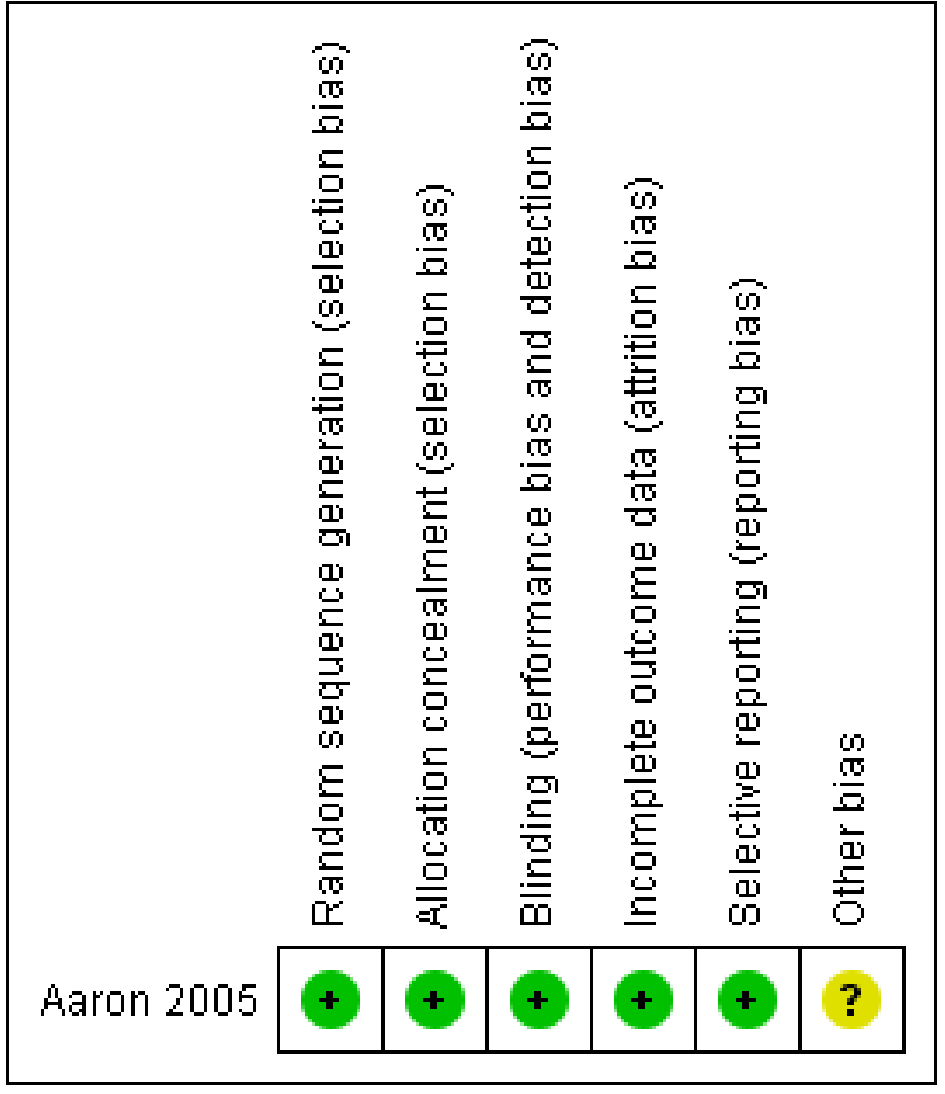

\section{Allocation}

Allocation of participants was done through a computer-generated random listing of the two treatment assignments blocked in groups of four and stratified by site; this was therefore graded as adequate. Randomisation was undertaken centrally through the research pharmacy. The research staff, participants and caregivers were unaware of the allocation. Thus the concealment of allocation was graded as adequate.

\section{Blinding}

The person responsible for participant care, the participant and the outcome assessor were all blinded.

\section{Incomplete outcome data}

All 132 participants receiving an intervention were included in the final analysis (intention-to-treat analysis) presented in the study report. There were no withdrawals from the study.

\section{Selective reporting}

Although the study itself reported all the data for all participants enrolled, we were only able to retrieve data for one study outcome (time to subsequent exacerbation) for participants infected with $P$ aeruginosa. However, this was the study's primary outcome and is a clinically relevant outcome listed in this review.

\section{Other potential sources of bias}

We judged there to be an unclear risk from other sources of bias for a number of reasons. In this study antibiotics were prescribed for participants randomised to the MCBT arm by one investigator, whereas antibiotics were prescribed for participants randomised to the control arm by the participants' own doctors. This approach was necessary, since the local physicians had to remain blinded to the MCBT results, but it could have affected the study outcomes. A second limitation is that this study was powered to show a $79 \%$ increase in the time to next exacerbation, but did not have the statistical power to exclude a smaller effect of MCBT-directed therapy. FInally, conventional clinical microbiological testing and MCBT testing both involve the culture of planktonically growing bacteria (i.e., free floating bacteria growing in broth). Bacteria growing in biofilms, e.g. $P$ aeruginosa, have been shown to be significantly more resistant to antimicrobials than those growing planktonically.

\section{Effects of interventions}

See: Summary of findings for the main comparison

\section{Primary outcomes}

\section{Lung function}

a. $\mathrm{FEV}_{\mathbf{1}}$ (L/min or \% predicted)

Data specific for the participants with only $P$ aeruginosa infection were not available for this outcome.

\section{b. FVC (L/min or \% predicted)}

Data specific for the participants with only $P$ aeruginosa infection were not available for this outcome.

Combination antimicrobial susceptibility testing for acute exacerbations in chronic infection of Pseudomonas aeruginosa in cystic 


\section{Time to next pulmonary exacerbation}

Seven participants (three in the MCBT group and four in the control group) did not have a subsequent pulmonary exacerbation during the study follow-up period (up to 4.5 years). Based on information sent to us directly from the study investigator, for the participants specifically infected with only $P$ aeruginosa, the hazard ratio for subsequent exacerbation was 0.82 for the conventional (control) group compared to the MCBT group ( $95 \% \mathrm{Cl} 0.44$ to 1.51$)(\mathrm{P}=0.52)$ (Aaron 2005).

\section{Secondary outcomes}

\section{Quality of life}

Although the included study measured dyspnoea using the transitional dyspnoea index score, the study did not have a validated measurement of quality of life (Aaron 2005).

\section{Length of hospital stay}

Data specific for the participants with only $P$ aeruginosa infection were not available for this outcome.

\section{Sputum bacterial density (CFU/mL)}

Data specific for the participants with only $P$ aeruginosa infection were not available for this outcome.

\section{Adverse events}

Data specific for the participants with only $P$ aeruginosa infection were not available for this outcome. However, the study did report serious adverse events for all participants. There were two serious adverse events (one case of allergic rash and one case of reversible hepatitis) in the MCBT group and seven serious adverse events in the control group (five cases of allergic rash and two deaths due to respiratory failure; $\mathrm{P}=0.17$ )

\section{Mortality}

Data specific for the participants with only $P$ aeruginosa infection were not available for this outcome. However, the study did report two deaths in the whole study cohort.

\section{DISCUSSION}

\section{Summary of main results}

The only included study showed that the use of multiple combination bactericidal antibiotic testing (MCBT) to guide antibiotic treatment of people with cystic fibrosis (CF) with acute pulmonary exacerbations due to Pseudomonas aeruginosa $(P$ aeruginosa) did not result in longer times to subsequent pulmonary exacerbations (Aaron 2005).

\section{Overall completeness and applicability of evidence}

It is important to note that the results of this study are based on one method of MCBT and they may not be generalizable to other methodologies such as checkerboard dilution assays. The primary outcome chosen by the investigators, time until the next pulmonary exacerbation, is highly clinically relevant to people with CF. However, the Aaron study was powered to detect a difference in this outcome for people with CF infected with several different types of multiresistant gram negative bacteria and not for people with CF infected with only P aeruginosa (subject of this review).
Any individual with CF who was chronically infected with multiresistant gram negative bacteria was eligible for enrolment into the study. However,in practice, MCBT tends to be reserved for those who fail empirically chosen antibiotic treatment or who have respiratory bacterial strains that are resistant to all antibiotics tested by conventional methods. It is possible that the use of MCBT may improve outcomes in this subset of people with CF.

In addition, testing antibiotics against bacteria grown planktonically, or "free-floating", in the laboratory may not accurately reflect the environment in the CF lung. For example, $P$ aeruginosa is known to grow as a biofilm, or a slime layer, in the sputum of people with CF (Drenkard 2002; Singh 2000). Antimicrobial susceptibility testing based on biofilm growth of $P$ aeruginosa may therefore be a more rational way of choosing antibiotics to treat pulmonary exacerbations and may lead to improved clinical outcomes in people with CF.

\section{Quality of the evidence}

The main strengths of the study were the quality of the methods (randomisation, allocation concealment and blinding) and the choice of a clinically relevant primary outcome.

If the antibiotics chosen based on the MCBT test were more effective than those chosen based on the conventional test, we would expect the pulmonary bacterial load to decrease more in the MCBT group than in the control group, leading to a longer time before a subsequent exacerbation (Regelmann 1990; Smith 1999). The Aaron study was powered to detect a difference in the time until the next pulmonary exacerbation in people with CF infected with several different types of multiresistant gram negative bacteria.

There were some limitations to the study. The study was only powered to show a minimum of $79 \%$ increase in the time to next exacerbation and did not have the power to detect a smaller increase in the time to next exacerbation. Given the multifactorial nature of pulmonary exacerbations in CF, it is unlikely that antibiotic choices alone could almost double the time to next exacerbation. Therefore, we cannot exclude a smaller effect of MCBT-guided therapy. Furthrmore, in the included study, one person prescribed antibiotics for the MCBT group while multiple individual physicians prescribed antibiotics for the control group. Although this was meant to simulate "real life" conditions, it introduces a significant degree of variability that the authors could not control for as there is no standardized method for choosing antibiotics. Choosing antibiotics based on their ability to kill bacteria in vitro may also not be as important as their potential anti-inflammatory effects. The study investigators correctly point out that the use of antibiotics such as azithromycin, which has no bactericidal effect against $P$ aeruginosa, but may have an anti-inflammatory effect, has been associated with clinical improvement in people with CF (Equi 2002; Saiman 2003).

Overall, we found the quality of the body of evidence to be moderate for the only outcome (time to next exacerbation) for which data were available for those people with infection due to $P$ aeruginosa; for other outcomes we were unable to judge the quality of the evidence as no data were available for the relevant subset of participants (Summary of findings for the main comparison). 


\section{Potential biases in the review process}

Overall, there was little risk of bias in the review process. We performed a comprehensive search of the literature not limited by language; we used broad search terms when searching the Cochrane CF clinical trials register, the clinicaltrials. gov website and the WHO trials website. The two authors independently assessed the studies, extracted the data, analysed the data and assessed the studies for bias.

\section{Agreements and disagreements with other studies or reviews}

There are no other studies or trials examining this particular question in CF. However, other studies that have examined the relationship between in vitro antimicrobial susceptibility testing and clinical outcomes in individuals with CF have similarly been unable to find a correlation. No study to date has identified a superior antibiotic regimen in the treatment of pulmonary exacerbations in people with CF, highlighting the multifactorial, complex nature of the infection and response to therapy.

\section{AUTHORS' CONCLUSIONS}

\section{Implications for practice}

The current evidence-base, limited to one study, is insufficient to determine the effect of choosing antibiotics based on combination antimicrobial susceptibility testing compared to choosing antibiotics based on conventional antimicrobial susceptibility testing in the treatment of acute pulmonary exacerbations and chronic infection in people with CF with $P$ aeruginosa.

\section{Implications for research}

A larger, adequately-powered, study is needed to determine if combination antimicrobial susceptibility testing may be beneficial in people with CF infected specifically with $P$ aeruginosa. A subgroup analysis could be undertaken on individuals who fail empirically chosen antibiotic treatment or who have respiratory bacterial strains that are resistant to all antibiotics tested by conventional methods. Such a study requires international collaboration in order to have sufficient power to detect a more modest improvement in a similar clinically relevant outcome.

\section{ACKNOWLEDGEMENTS}

The authors acknowledge the kind assistance of Dr Shawn Aaron who provided us with the data specific for participants in his study infected with only P aeruginosa.

This project was supported by the National Institute for Health Research, via Cochrane Infrastructure funding to the Cochrane Cystic Fibrosis and Genetic Disorders Group. The views and opinions expressed therein are those of the authors and do not necessarily reflect those of the Systematic Reviews Programme, NIHR, NHS or the Department of Health. 


\section{RE F E R E N C E S}

\section{References to studies included in this review}

Aaron 2005 \{published and unpublished data\}

Aaron S. Clinical evidence for combination antibiotic susceptibility testing (synergy testing) [abstract]. Pediatric Pulmonology 2008;43(Suppl 31):157. [CFGD Register: PI198c]

Aaron S, Vandemheen K, Ferris W, Tullis E, Haase D, Berthiaume Y, et al. Treatment of CF exacerbations based on multiple combination antibiotic susceptibility testing-a randomized, double-blind, controlled clinical trial. Pediatric Pulmonology 2005;40(Suppl 28):304. [CFGD Register: PI198a]

* Aaron SD, Vandemheen KL, Ferris W, Fergusson D, Tullis E, Haase D, et al. Combination antibiotic susceptibility testing to treat exacerbations of cystic fibrosis associated with multiresistant bacteria: a randomised, double-blind, controlled clinical trial. Lancet 2005;366(9484):463-71. [CFGD Register: Pl198b]

\section{References to studies excluded from this review}

\section{Moskowitz 2011 \{published data only\}}

Moskowitz SM, Emerson JC, McNamara S, Shell RD, Orenstein DM, Rosenbluth $\mathrm{D}$, et al. Randomized trial of biofilm testing to select antibiotics for cystic fibrosis airway infection. Pediatric Pulmonology 2011;46(2):184-92. [CFGD Register: PI245]

\section{Oermann 2013 \{published data only\}}

Oermann CM, McCoy KS, Retsch-Bogart GZ, Gibson R, McKevitt M, Montgomery B. Antibiotic susceptibility in Pseudomonas Aeruginosa (PA) isolates following repeated exposure to aztreonam for inhalation solution (AZLI) in patients with cystic fibrosis [abstract]. Pediatric Pulmonology 2009;44(Suppl 32):309, Abstract no: 278.

Oermann CM, McCoy KS, Retsch-Bogart GZ, Gibson R, McKevitt M, Montgomery B. Effect of repeated exposure to aztreonam for inhalation solution (AZLI) therapy on cystic fibrosis respiratory pathogens [abstract]. Pediatric Pulmonology 2009;8(Suppl 2):335, Abstract no: 353.

Oermann CM, McCoy KS, Retsch-Bogart GZ, Gibson RL, Montgomery AB. Effect of multiple courses of Aztreonam Lysine for inhalation (AZLI) on FEV1 and weight in patients with cystic fibrosis (CF) and Pseudomonas aeruginosa (PA): analysis of 18 month data from CP-AI-006 [abstract]. Journal of Cystic Fibrosis 2009;8(Suppl 2):S28, Abstract no: 107.

Oermann CM, McCoy KS, Retsch-Bogart GZ, Gibson RL, Quittner AL, Montgomery AB. Adherence over multiple courses of Aztreonam for inhalation (AZLI): effect on diseaserelated endpoints in patients with cystic fibrosis (CF) and Pseudomonas aeruginosa (PA) [abstract]. Journal of Cystic Fibrosis 2009;8(Suppl 2):S28, Abstract no: 109.

* Oermann CM, Retsch-Bogart GZ, Quittner AL, Gibson RL, McCoy KS, Montgomery AB, et al. An 18-month study of the safety and efficacy of repeated courses of inhaled aztreonam lysine in cystic fibrosis. Pediatric Pulmonology 2010; Vol. 45, issue 11:1121-34. []

\section{Wainwright 2011 \{published data only\}}

Wainwright C, Nakamura C, Geller D, Montgomery AB. A doubleblind, multinational, randomized, placebo-controlled trial evaluating aztreonam for inhalation solution (AZLI) in patients with cystic fibrosis (CF), mild lung disease and $P$. aeruginosa. Journal of Cystic Fibrosis 2010;9(Suppl 1):S22, Abstract no: 81. [CFGD Register: PI243a]

Wainwright CE, Quittner AL, Geller DE, Nakamura C, Wooldridge JL, Gibson RL, et al. Aztreonam for inhalation solution (AZLI) in patients with cystic fibrosis, mild lung impairment, and P. aeruginosa. Journal of Cystic Fibrosis 2011;10(4):234-42. [CFGD Register: PI243b]

\section{Waters 2010 \{published data only\}}

Ratjen F, Stanojevic S, Sonneveld N, Grasemann H, Yau Y, Tullis E, et al. Predictors of response to antibiotic treatment of pulmonary exacerbations in cystic fibrosis patients [abstract]. Pediatric Pulmonology 2014;49 Suppl 38:355, Abstract no: 384. [CENTRAL: 1012525; CFGD Register: PI244c; CRS: 5500131000000179]

Waters V, Ratjen F, Tullis E, Corey M, Matukas L, Leahy R, et al. Randomized double blind controlled trial of the use of a biofilm antimicrobial susceptibility assay to guide antibiotic therapy in chronic pseudomonas aeruginosa infected cystic fibrosis patients [abstract]. Pediatric Pulmonology 2010;45 Suppl 33(Suppl 33):339, Abstract no: 311. [CENTRAL: 848814; CFGD Register: PI244a; CRS: 5500100000004249$]$

Waters V, Yau Y. Use of a biofilm antimicrobial susceptibility assay to guide antibiotic therapy. //clinicaltrials.gov/show/ NCT00786513 2015. [CENTRAL: 1050336; CFGD Register: PI244g; CRS: 5500131000000354]

Waters VJ, Ratjen F, Tullis E, Wilcox PG, Freitag A, Chilvers M, et al. Randomized controlled trial of biofilm antimicrobial susceptibility testing in pulmonary exacerbations in cystic fibrosis patients with chronic pseudomonas aeruginosa infection [abstract]. Pediatric Pulmonology 2014;49 Suppl 38:319, Abstract no: 287. [CFGD Register: PI244b; CRS: 5500131000000178]

Waters VJ, Stanojevic S, Sonneveld N, Klingel M, Grasemann H, Yau YC, et al. Factors associated with response to treatment of pulmonary exacerbations in cystic fibrosis patients. Journal of Cystic Fibrosis : Official Journal of the European Cystic Fibrosis Society 2015;14(6):755-62. [CFGD Register: PI244h; CRS: 5500135000001435; PUBMED: 25690407]

Yau YCW, Ratjen F, Tullis E, Wilcox P, Freitag A, Chilvers M, et al. Online supplement to "Randomized controlled trial of biofilm antimicrobial susceptibility testing in cystic fibrosis patients". Journal of Cystic Fibrosis : Official Journal of the European Cystic Fibrosis Society 2015;14:1-13 online. [CENTRAL: 1050334; CFGD Register: PI244e; CRS: 5500131000000352] 
Yau YCW, Ratjen F, Tullis E, Wilcox P, Freitag A, Chilvers M, et al. Online supplementary tables from "Randomized controlled trial of biofilm antimicrobial susceptibility testing in cystic fibrosis patients". Journal of Cystic Fibrosis : Official Journal of the European Cystic Fibrosis Society 2015;14:1-4 online. [CENTRAL: 1050335; CFGD Register: PI244f; CRS: 5500131000000353]

Yau YCW, Ratjen F, Tullis E, Wilcox P, Freitag A, Chilvers M, et al. Randomized controlled trial of biofilm antimicrobial susceptibility testing in cystic fibrosis patients. Journal of Cystic Fibrosis: Official Journal of the European Cystic Fibrosis Society 2015;14:262-6. [CENTRAL: 1015278; CFGD Register: PI244d; CRS: $5500131000000261]$

\section{Additional references}

\section{Aaron 2000}

Aaron SD, Ferris W, Henry DA, Speert DP, Macdonald NE. Multiple combination bactericidal antibiotic testing for patients with cystic fibrosis infected with Burkholderia cepacia. American Journal of Respiratory and Critical Care Medicine 2000;161(4 Pt 1):1206-12.

\section{Burns 2001}

Burns JL, Gibson RL, McNamara S, Yim D, Emerson J, Rosenfeld M, et al. Longitudinal assessment of Pseudomonas aeruginosa in young children with cystic fibrosis. Journal of Infectious Diseases 2001;183(3):444-52.

\section{Ceri 1999}

Ceri H, Olson ME, Stremick C, Read RR, Morck D, Buret A. The Calgary Biofilm Device: new technology for rapid determination of antibiotic susceptibilities of bacterial biofilms. Journal of Clinical Microbiology 1999;37(6):1771-6.

\section{Donner 2001}

Donner A, Piaggio G, Villar J. Statistical methods for the metaanalysis of cluster randomized trials. Statistical Methods in Medical Research 2001;10(5):325-38.

\section{Drenkard 2002}

Drenkard E, Ausubel FM. Pseudomonas biofilm formation and antibiotic resistance are linked to phenotypic variation. Nature 2002;416(6882):740-3.

\section{Equi 2002}

Equi A, Balfour-Lynn IM, Bush A, Rosenthal M. Long term azithromycin in children with cystic fibrosis: a randomised, placebo-controlled crossover trial. Lancet 2002;360:978-84.

\section{Foweraker 2005}

Foweraker JE, Laughton CR, Brown DF, Bilton D. Phenotypic variability of Pseudomonas aeruginosa in sputa from patients with acute infective exacerbation of cystic fibrosis and its impact on the validity of antimicrobial susceptibility testing. Journal of Antimicrobial Chemotherapy 2005;55(6):921-7.

\section{Gaillard 1995}

Gaillard JL, Cahen P, Delacourt C, Silly C, Le Bourgeois M, Coustère $\mathrm{C}$, et al. Correlation between activity of beta-lactam agents in vitro and bacteriological outcome in acute pulmonary exacerbations of cystic fibrosis. European Journal of Clinical Microbiology \& Infectious Diseases: official publication of the European Society of Clinical Microbiology 1995;14(4):291-6.

\section{Gibson 2003}

Gibson RL, Burns JL, Ramsey BW. Pathophysiology and management of pulmonary infections in cystic fibrosis. American Journal of Respiratory and Critical Care Medicine 2003;168(8):918-51.

\section{Henry 1992}

Henry RL, Mellis CM, Petrovic L. Mucoid Pseudomonas aeruginosa is a marker of poor survival in cystic fibrosis. Pediatric Pulmonology 1992;12(3):158-61.

\section{Higgins 2003}

Higgins JPT, Thompson SG, Deeks JJ, Altman DG. Measuring inconsistency in meta-analyses. BMJ 2003;327(7414):557-60.

\section{Higgins 2011}

Higgins JP, Green S, editor(s). Cochrane Handbook for Systematic Reviews of Interventions Version 5.1.0 (updated March 2011). The Cochrane Collaboration, 2011. Available from handbook.cochrane.org.

\section{Jüni 2001}

Jüni P, Altman DG, Egger M. Systematic reviews in health care: Assessing the quality of controlled clinical trials. BMJ 2001;323(7303):42-6.

\section{Kosorok 2001}

Kosorok MR, Zeng L, West SE, Rock MJ, Splaingard ML, Laxova A, et al. Acceleration of lung disease in children with cystic fibrosis after Pseudomonas aeruginosa acquisition. Pediatric Pulmonology 2001;32(4):277-87.

\section{Lee 2003}

Lee TWR, Brownlee KG, Conway SP, Denton M, Littlewood JM. Evaluation of a new definition for chronic Pseudomonas aeruginosa infection in cystic fibrosis patients. Journal of Cystic Fibrosis 2003;2(1):29-34

\section{Moskowitz 2004}

Moskowitz SM, Foster JM, Emerson J, Burns JL. Clinically feasible biofilm susceptibility assay for isolates of Pseudomonas aeruginosa from patients with cystic fibrosis. Journal of Clinical Microbiology 2004;42(5):1915-22.

\section{Moskowitz 2005}

Moskowitz SM, Foster JM, Emerson JC, Gibson RL, Burns JL. Use of Pseudomonas biofilm susceptibilities to assign simulated antibiotic regimens for cystic fibrosis airway infection. Journal of Antimicrobial Chemotherapy 2005;56(5):879-86.

\section{Pamukcu 1995}

Pamukcu A, Bush A, Buchdahl R. Effects of pseudomonas aeruginosa colonization on lung function and anthropometric variables in children with cystic fibrosis. Pediatric Pulmonology 1995;19(1):10-5.

Combination antimicrobial susceptibility testing for acute exacerbations in chronic infection of Pseudomonas aeruginosa in cystic 


\section{Parmar 1998}

Parmar MK, Torri V, Stewart L. Extracting summary statistics to perform meta-analyses of the published literature for survival endpoints. Statistics in Medicine 1998;17(24):2815-34.

\section{Prince 2002}

Prince AS. Biofilms, antimicrobial resistance, and airway infection. The New England Journal of Medicine 2002;347(14):1110-1.

\section{Regelmann 1990}

Regelmann WE, Elliott GR, Warwick WJ, Clawson CC. Reduction of sputum Pseudomonas aeruginosa density by antibiotics improves lung function in cystic fibrosis more than do bronchodilators and chest physiotherapy alone. American Review of Respiratory Disease 1990;141(4 Pt 1):914-21.

\section{Rosenfeld 2001}

Rosenfeld M, Emerson J, Williams-Warren J, Pepe M, Smith A Montgomery $\mathrm{AB}$, et al. Defining a pulmonary exacerbation in cystic fibrosis. Journal of Pediatrics 2001;139(3):359-65.

\section{Saiman 1996}

Saiman L, Mehar F, Niu WW, Neu HC, Shaw KJ, Miller G, Prince A. Antibiotic susceptibility of multiply resistant Pseudomonas aeruginosa isolated from patients with cystic fibrosis, including candidates for transplantation. Clinical infectious diseases : an official publication of the Infectious Diseases Society of America 1996;23(3):532-7.

\section{Saiman 2003}

Saiman L, Marshall BC, Mayer-Hamblett N, et al. Azithromycin in patients with cystic fibrosis chronically infected with Pseudomonas aeruginosa: a randomized controlled trial. JAMA 2003;290:1749-56.

\section{CHARACTERISTICS OF STUDIES}

Characteristics of included studies [ordered by study ID]

\section{Singh 2000}

Singh PK, Schaefer AL, Parsek MR, Moninger TO, Welsh MJ, Greenberg EP. Quorum-sensing signals indicate that cystic fibrosis lungs are infected with bacterial biofilms. Nature 2000;407(6805):762-4.

\section{Smith 1999}

Smith AL, Doershuk C, Goldman D, Gore E, Hilman B, Marks M, et al. Comparison of a B-lactam alone versus B-lactam and an aminoglycoside for a pulmonary exacerbation in cystic fibrosis. Journal of Pediatrics 1999;134(4):413-21.

\section{Smith 2003}

Smith AL, Fiel SB, Mayer-Hamblett N, Ramsey B, Burns JL. Susceptibility testing of Pseudomonas aeruginosa isolates and clinical response to parenteral antibiotic administration: lack of association in cystic fibrosis. Chest 2003;123(5):1495-502.

\section{References to other published versions of this review}

\section{Waters 2008}

Waters V, Ratjen F. Antimicrobial susceptibility testing for acute exacerbations in chronic infection of Pseudomonas aeruginosa in cystic fibrosis. Cochrane Database of Systematic Reviews 3, Issue 2008. [DOI: 10.1002/14651858.CD006961]

\section{Waters 2015}

Waters V, Ratjen F. Combination antimicrobial susceptibility testing for acute exacerbations in chronic infection of Pseudomonas aeruginosa in cystic fibrosis. Cochrane Database of Systematic Reviews 2015, Issue 11. [DOI: 10.1002/14651858.CD006961.pub3]

* Indicates the major publication for the study

\section{Aaron 2005}

\section{Methods}

Randomised, double-blind controlled trial.

Parallel design.

Duration: 14 days treatment, follow up every 3 months for up to 4.5 years.

Location: multicentre, 10 sites in Canada and 2 in Australia. cia complex, Pseudomonas aeruginosa, Stenotrophomonas maltophilia, or Achromobacter xylosoxidans bacteria (at least 2 sputum cultures within the past 12 months that had grown these organisms).

132 participants with acute pulmonary exacerbations of CF (defined according to criteria published by the 1994 Cystic Fibrosis Foundation Microbiology and Infectious Disease Consensus Conference) infected with multiresistant bacteria randomised on admittance for treatment.

\section{MCBT group}

$\mathrm{n}=64,43$ (67.2\%) infected with Pseudomonas aeruginosa. 
Aaron 2005 (Continued)

Age (mean (SD)): 29.5 (8.2) years.

Gender: 29 males, 35 females.

FEV $_{1} \%$ predicted (mean (SD)): $44 \cdot 0$ (16.4)\%.

Diabetes: 15 (23.4\%).

Pancreatic insufficiency: 63 (98.4\%).

Liver disease: 6 (9.4\%).

\title{
Conventional treatment group
}

$\mathrm{n}=68,39(57.4 \%)$ infected with Pseudomonas aeruginosa

Age (mean (SD)): $25.8(6.5)$ years.

Gender: 31 males, 37 females.

FEV $_{1} \%$ predicted (mean (SD)): $39.1(16.7) \%$.

Diabetes: 13 (19.1\%).

Pancreatic insufficiency: 65 (95.6\%).

Liver disease: 8 (11.8\%).

\begin{abstract}
Interventions 14-day course of any two IV antibiotics chosen on the basis of MCBT or control (separate testing).
IV aminoglycosides were given once daily at 2 study sites, $2 x$ daily at 1 site, and thrice daily at the 9 other sites. In each case MCBT orders, and control orders, conformed to the local centre's treatment practice.
\end{abstract}

\begin{tabular}{|c|c|}
\hline Outcomes & $\begin{array}{l}\text { Lung function (FEV }{ }_{1} \text { and } \mathrm{FVC} \text { ), time to next pulmonary exacerbation, length of hospital stay, sputum } \\
\text { bacterial density, adverse events, mortality, dyspnoea, treatment failures, white blood cell counts, C- } \\
\text { reactive protein concentrations in serum, and erythrocyte sedimentation rates, compliance. }\end{array}$ \\
\hline
\end{tabular}

Notes

Study sample size was calculated based on expected median survival times to next exacerbation; 132 participants were randomised, resulting in $83 \%$ power to show a difference between the two treatment groups.

The study sponsors had no role in study design, data collection, data analysis, data interpretation, or writing of the report. The corresponding author had full access to all the data in the study and had final responsibility for the decision to submit for publication.

\section{Risk of bias}

\section{Bias Authors' judgement Support for judgement}

Random sequence genera- Low risk Randomisation was done centrally through the research pharmacy using tion (selection bias) a computer-generated random listing of the two treatment assignments blocked in groups of four and stratified by site.

Allocation concealment Low risk

The research staff, participants and caregivers were unaware of the allocation.

(selection bias)

The principal investigator in Ottawa (SDA) was the only physician with access to the MCBT test results and he ordered the MCBT-directed therapy for all participants. The research pharmacist at each hospital was the only member of the investigative team aware of the randomisation assignment,

\begin{tabular}{ll}
\hline $\begin{array}{l}\text { Blinding (performance } \\
\text { bias and detection bias) }\end{array}$ & Low risk
\end{tabular}$\quad \begin{aligned} & \text { Each hospital pharmacist prepared the two blinded IV antibiotics for ad- } \\
& \text { ministration (labelled "antibiotic \#1" and "antibiotic \#2") and also prepared }\end{aligned}$

Combination antimicrobial susceptibility testing for acute exacerbations in chronic infection of Pseudomonas aeruginosa in cystic 
Aaron 2005 (Continued)

All outcomes nebulised tobramycin, or nebulised identical placebo tobramycin, for each randomised patient. Blinded study drugs were administered on the hospital wards, or in some cases, in the participants' homes under supervision by home-care nursing staff.
Incomplete outcome data Low risk (attrition bias)

All outcomes
All 132 participants receiving an intervention were included in the final analysis (intention-to-treat analysis). There were no withdrawals from the study.

\section{Selective reporting (re- Low risk} porting bias)
Although the study itself reported all the data for all participants enrolled ( $\mathrm{n}$ = 132), we were only able to retrieve 1 study outcome (time to subsequent exacerbation) for participants infected with $P$ aeruginosa. However, this was the study's primary outcome and is a clinically relevant outcome to this review.

A potential limitation of this study is that antibiotics were prescribed for participants randomised to the MCBT arm by one investigator, whereas antibiotics were prescribed for participants randomised to the control arm by the participants' own doctors. This approach was necessary, since the local physicians had to remain blinded to the MCBT results, but it could have affected the study outcomes.

A second limitation is that this study was powered to show a $79 \%$ increase in the time to next exacerbation and did not have the statistical power to exclude a smaller effect of MCBT-directed therapy.

Conventional clinical microbiological testing, and MCBT testing, involves the culture of planktonically growing bacteria (i.e. free floating bacteria growing in broth). Bacteria growing in biofilms, e.g. Pseudomonas aeruginosa, have been shown to be significantly more resistant to antimicrobials than those growing planktonically.

CF: cystic fibrosis

$\mathrm{FEV}_{1}$ : forced expiratory volume in one second

FVC: forced vital capacity

IV: intravenous

MCBT: multiple combination bactericidal antibiotic testing

SD: standard deviation

Characteristics of excluded studies [ordered by study ID]

\begin{tabular}{ll}
\hline Study & Reason for exclusion \\
\hline Moskowitz 2011 & A trial of biofilm antimicrobial susceptibility testing. \\
\hline Oermann 2013 & Not a trial of combination antimicrobial susceptibility testing. \\
\hline Wainwright 2011 & Not a trial of combination antimicrobial susceptibility testing. \\
\hline Waters 2010 & A trial of biofilm antimicrobial susceptibility testing. \\
\hline
\end{tabular}

\section{APPENDICES}

\section{Appendix 1. Search strategy: Clinicaltrials.gov (searched 08 March 2017)}




\begin{tabular}{ll}
\hline Search 1 & CF AND antimicrobial susceptibility \\
\hline
\end{tabular}

Search $2 \quad$ CF AND antibiotics

Appendix 2. Search Strategy: apps.who.int/trialsearch/ (searched 08 March 2017)

\begin{tabular}{ll}
\hline Search 1 & CF AND antimicrobial susceptibility \\
\hline Search 2 & CF AND antibiotics \\
\hline
\end{tabular}

WHAT'S NEW

\begin{tabular}{lll}
\hline Date & Event & Description \\
\hline 19 March 2020 & Amended & $\begin{array}{l}\text { Clarification statement added from Alan Smyth, Co-ordinating } \\
\text { Editor on 19 March 2020: This review was found by the Cochrane }\end{array}$ \\
& $\begin{array}{l}\text { Funding Arbiters, post-publication, to be noncompliant with the } \\
\text { Cochrane conflict of interest policy, which includes the relevant } \\
\text { parts of the Cochrane Commercial Sponsorship Policy. The re- } \\
\text { view will be updated by April 2020; the authors of the future up- } \\
\text { date will be free of conflicts. }\end{array}$ \\
\hline
\end{tabular}

\section{HIST O R Y}

Protocol first published: Issue 1, 2008

Review first published: Issue 3, 2008

\begin{tabular}{lll}
\hline Date & Event & Description \\
\hline 4 May 2017 & New search has been performed & $\begin{array}{l}\text { A search of the Cystic Fibrosis and Genetic Disorders Trials Reg- } \\
\text { ister identified seven new references which were additional ref- } \\
\text { erences to two previously excluded studies (Waters 2010; Wain- } \\
\text { wright 2011). }\end{array}$
\end{tabular}

A summary of findings table has been added to the review.

\begin{tabular}{lll}
\hline 4 May 2017 & $\begin{array}{l}\text { New citation required but conclusions } \\
\text { have not changed }\end{array}$ & $\begin{array}{l}\text { No new data have been added to the review and our conclusions } \\
\text { remain the same. }\end{array}$ \\
\hline 10 August 2015 New search has been performed & $\begin{array}{l}\text { The only trial listed in Included studies in the review was pub- } \\
\text { lished in 2005, and we have not identified any further relevant } \\
\text { trials up to July 2015. We will continue to run searches to identi- } \\
\text { fy any potentially relevant trials; however, we do not plan to up- } \\
\text { date other sections of the review until new trials are published. }\end{array}$ \\
$\begin{array}{l}\text { A search of the Cystic Fibrosis and Genetic Disorders Group's Cys- } \\
\text { tic Fibrosis Trials Register did not identify any new potentially } \\
\text { relevant references for inclusion in this review. }\end{array}$
\end{tabular}

Combination antimicrobial susceptibility testing for acute exacerbations in chronic infection of Pseudomonas aeruginosa in cystic 


\begin{tabular}{lll}
\hline Date & Event & Description \\
\hline 10 August 2015 & $\begin{array}{l}\text { New citation required but conclusions } \\
\text { have not changed }\end{array}$ & $\begin{array}{l}\text { No new trials have been included in the review, therefore our } \\
\text { conclusions remain the same. }\end{array}$ \\
\hline 8 July 2013 & Review declared as stable & $\begin{array}{l}\text { The only trial listed in Included studies in the review was pub- } \\
\text { lished in 2005, and we have not identified any further relevant } \\
\text { trials up to September 2011; although in July 2013 the full pa- } \\
\text { per to an already excluded trial was published and the reference } \\
\text { has now been added. We therefore do not plan to update this re- } \\
\text { view until new trials are published, although we will search the } \\
\text { Group's Cystic Fibrosis Trials Register on a two-yearly cycle. }\end{array}$ \\
\hline
\end{tabular}

28 September $2011 \quad$ New search has been performed

A search of the Group's Cystic Fibrosis Register identified six references to three studies. Four of these were additional references to an already excluded study (Oermann 2013) and the other two references have also been excluded (Moskowitz 2011; Waters 2010).

The only trial listed in Included studies in the review was published in 2005, and we have not identified any further relevant trials up to September 2011. We therefore do not plan to update this review until new trials are published, although we will search the Group's Cystic Fibrosis Trials Register on a two-yearly cycle.
A search of the Group's Cystic Fibrosis Register identified two new references; one was an additional reference to an already included study (Aaron 2005) and the other study was excluded (Oermann 2013). A search of www.clinicaltrials.gov did not identify any new references.

8 May $2008 \quad$ Amended Converted to new review format.

\section{CONTRIBUTIONS OF AUTHORS}

The review was conceived by Dr Valerie Waters. Dr Waters drafted the protocol with comments from Professor Felix Ratjen.

Dr Waters acts as guarantor of the review.

\section{DECLARATIONS OF INTEREST}

None known.

Clarification statement added from Alan Smyth, Co-ordinating Editor on 19 March 2020: This review was found by the Cochrane Funding Arbiters, post-publication, to be noncompliant with the Cochrane conflict of interest policy, which includes the relevant parts of the Cochrane Commercial Sponsorship Policy. The review will be updated by April 2020; the authors of the future update will be free of conflicts.

\section{SOURCES OF SUPPORT}

\section{Internal sources}

- No sources of support supplied

\section{External sources}

- National Institute for Health Research, UK.

This systematic review was supported by the National Institute for Health Research, via Cochrane Infrastructure funding to the Cochrane Cystic Fibrosis and Genetic Disorders Group. 


\section{DIFFERENCES BETWEEN PROTOCOLAND REVIEW}

In 2017 a summary of findings table was added in line with guidance from Cochrane.

\section{N DEX TERMS}

\section{Medical Subject Headings (MeSH)}

${ }^{*}$ Pseudomonas aeruginosa [drug effects]; Anti-Bacterial Agents [*therapeutic use]; Chronic Disease; Cystic Fibrosis [ ${ }^{*}$ microbiology]; Disease Progression; Drug Therapy, Combination; Microbial Sensitivity Tests; Pseudomonas Infections [ ${ }^{*}$ drug therapy]; Randomized Controlled Trials as Topic

\section{MeSH check words}

Humans 